\title{
Dynamical explorations of nuclear structures in barred galaxies
}

\author{
J. Anosova and G. F. Benedict \\ University of Texas at Austin, Austin, TX 78712, USA
}

\begin{abstract}
.
We construct models of a galaxy in order to provide a possible identification of the dynamical processes that lead to the formation of structure observed in galactic nuclei. We assume that the center of our model contains a very massive double black hole, surrounded by relatively lowmass particles - star clusters, gas, and dust complexes. Our previous work (Anosova et al. 1994, 1995) showed that the dynamical evolution of such a model produces many structures similar to those observed in the nuclei of galaxies, including rings and various types of flows and jets. In such models the 'gravitational slingshot' effect frequently occurs.

We consider a number of such models with different initial parameters. Comparison of our models with the observed structure of NGC 4314 shows good agreement at one stage of the evolution for certain combinations of initial parameters. The model predicts the velocity field observed in NGC 4314.
\end{abstract}

\section{Introduction}

About $50 \%$ of disk galaxies have a central bar in the disk plane (Binney and Tremaine, 1987).

Bars can be found in all types of disk galaxies, from the earliest to the latest stages of the Hubble sequence. A barred galaxy may also contain a spheroidal bulge at its centre, spirals in the outer and inner disc and other features, including multiple nuclei, inner and outer rings and lenses. Symmetry and asymmetry in both the light and kinematics are quite common. The velocities in the barred region manifest strong non-circular streaming motions, with the major axes of the orbits elongated along the bar.

Most dynamical studies of these complicated systems by computer simulations have utilised hydrodynamics, gravitational potential theory, stellar orbital theory, as well as the gravitational N-body problem with large $\mathrm{N}$ (see Combes 1993, Sellwood 1987, 1993; Binney and Tremaine 1987 and references therein, and Yoshiaki and Keiichi 1996). All these dynamical studies have assumed collisionless systems. But we are still far from a complete understanding of the dynamical structure and the kinematics of barred galaxies and their features.

Anosova and Anandarao (1994) and Anosova and Tanikawa (1995) showed that various structures observed in celestial objects can be generated by simply assuming the existence of two dominating centers of attraction rather than one. 
In this work we have studied the dynamical evolution of galaxy models with central massive binaries (black holes) surrounded by relatively low-mass particles that could be star clusters or gas and dust complexes. We carried out computer simulations in the framework of the N-body problem, taking into account strong interactions of bodies. We studied the dynamics of these models, considering the general three-body problem with numerous initial conditions.

This problem can be compared with that studied by Murai and Fujimoto (1980) and Lin and Lynden-Bell (1982) in which they have considered the dynamical models of the Magellanic Stream in the triple galaxy system consisting of our Galaxy and the two Magellanic Clouds. They have examined the dynamics of this triple system with many zero-mass particles in the LMC and have studied the process of the formation of the flow (the Magellanic Stream) of zero-mass particles from the LMC.

Valtonen (1984a, b; 1985), and Valtonen and Byrd $(1979,1986)$ used a similar method (a compilation of many initial conditions for the restricted three-body problem) in order to study the process of dynamical formation of structures of galaxy clusters with massive centrally located binary galaxies. They showed that structure in their modelled systems is sometimes similar to observed structures of galaxy clusters (for example, the Coma Cluster).

Toomre and Toomre (1972) simulated the formation of bridges and tails due to the parabolic passage of a perturber by a galaxy surrounded by zeromass particles. Lin and Saslaw (1977) considered the flyby of a single star and a binary with associated disks of massless particles to explain the formation of double radio sources. Basu et al. (1993) modeled extragalactic jets by mergers of binary black holes surrounded by accretion disks. In these restricted three-body or four body investigations, the motions of main bodies are either parabolic, hyperbolic, or highly elliptic.

Recently Yoshiaki and Keiichi (1996) have considered a hydrodynamical model consisting of the merger of a single massive black hole with a nuclear gas disk containing a super-massive black hole in the center. A black hole binary forms quickly. An asymmetrical spiral pattern then appears. The masses of the two black holes are different. The authors used a collisionless model of a disk galaxy, and, of course, could not consider the strong interactions of particles and their trajectories.

\section{Description of the present model}

Anosova et al. $(1994,1995)$ and we in this work consider a central massive binary with a circular orbit. This is a long-lived binary system. Low-mass particles may interact with this binary nucleus many times during the dynamical evolution of the entire system.

In such models the 'gravitational slingshot' effect (Agekian and Anosova 1967, 1991, Anosova 1986, 1991; Valtonen 1988, Anosova and Zavalov 1989) occurs many times, especially during the total collapse of a system.

We construct dynamical models to identify possible processes that lead to the formation of structure observed in the nucleus of NGC 4314. Because the structure of this galaxy is symmetrical, the central massive binary must have components with equal masses (Anosova et al. 1994, 1995). 
We carried out our calculations in the framework of the three-body problem with 100,000 initial configurations. Our model consists of a central, massive binary $M_{1}, M_{2}$ with a circular orbit and a number of low-mass particles $M_{i}$ on several shells with different radii and concentric to the center of gravity of the binary. The particle shells together form a spherical system with a massive point-mass binary inside. Most of the shell particles are initially beyond the central binary orbit, while some are inside the orbit.

We modifed our code (based on the code 'TRIPLE' by Aarseth and Zare, 1974) to allow us to fix results of the integration of the general three-body problem while considering several thousand initial conditions simultaneously. Our model permits us to inspect structures in the models in 6-dimensional coordinate and velocity space. We also study the effects of projection in coordinate and velocity space. Multiple runs of the model allow us to classify the strong triple interactions of bodies resulting in various structures of models.

Our dynamical system of units are : for the distances, the semi-major axis $a_{o}$ of the binary; and for the time, the period $P_{o}$ of the binary; $G=1$. In this system the binary mass $M_{1}+M_{2}=4 \pi^{2}$; the ratio $M_{i} / M_{1}=100, M_{1}=M_{2}$; the initial parameters $a_{o}, e_{o}$ and $P_{o}$ of the central massive binary orbit do not change in time.

For each combination of parameters, we carry out a numerical integration spanning a time duration $T_{e v}=40 P_{o}$; for several cases $T_{e v}=100 P_{o}$.

In our models we often observe nearly simultaneous approaches of small-mass bodies to the center of mass of the binary. As a consequence most low-mass particles escape from the system. Before escaping, these particles form flows like open spirals. Particles with lower velocities return to the center of a system. Then, flows with the appearance of jets, rings and closed spirals form. Particles which initially were inside the binary orbit or close to it, form a stable central dumbbell bar (see Fig. 1).

\section{Results - comparison with NGC $\mathbf{4 3 1 4}$}

\subsection{Observations of NGC 4314 .}

From Hubble Space Telescope observations we have obtained a higher-resolution view of the complex nuclear morphology of the galaxy NGC 4314 (Benedict et al. 1992, 1993, 1996) - see Figs. 2a, 5a.

NGC 4314 is an anemic barred galaxy with recent star formation confined to a nuclear ring. Observations resolve the nuclear ring into multiple sites of new star formation and resolve the associated dust lanes into discrete clouds. In the center we see a nuclear bar with newer stars near its ends. We also resolve several star clusters associated with $\mathrm{H}$ II regions in the nuclear ring. The HST data provide many details in distributions of stars, dust, and gas in and near the nuclear ring. The nucleus contains no extreme concentration of stars. Massive star formation is confined to a ring and star production is episodic, because the ring is not uniformly packed with new stars. 


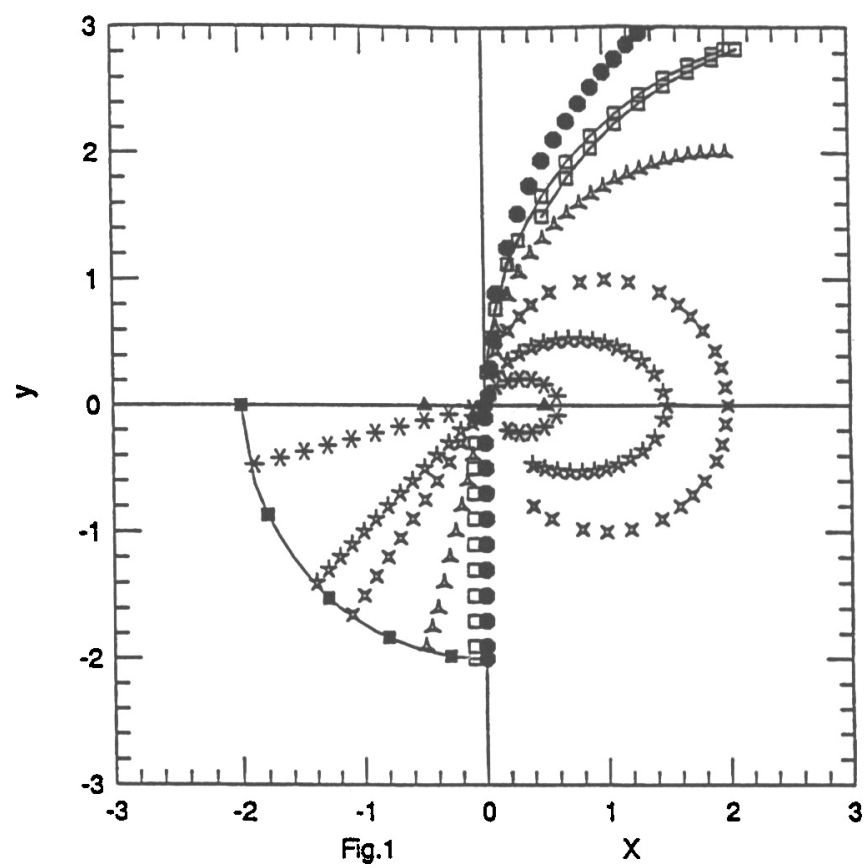

Figure 1. Basic Dynamical mechanisms for the formation of various nuclear jets and flows caused by strong interactions with a binary nucleus; the trajesctories of small mass bodies during strong interactions with the center of mass of the system. The filled cicles show the trajectories of escaping bodies; the open squares the motions of bodies that return to the center of inertia of a system after a failed ejection. Other symbols show trajectories of bodies that form different types of large and small rings inside a system. A part of the large circle in the negative quadrant $(x<0, y<0)$ shows the initialpositions of the small-mass particles on a shell around a massive binary. The filled squares show schematically the regions of initial positions of particles on a shell with different kinds of final motions: 

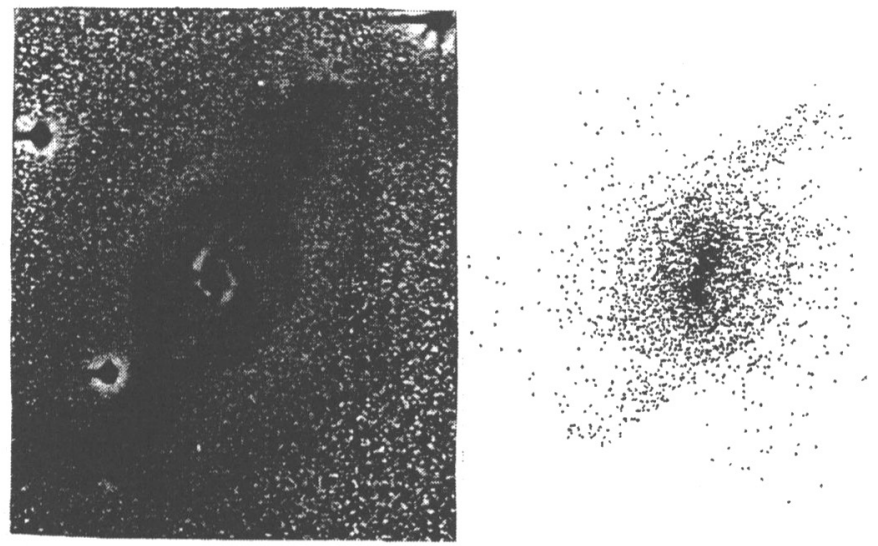

Fig. 2a

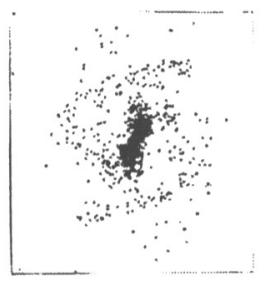

Fig. $2 c$
Fig. 2b

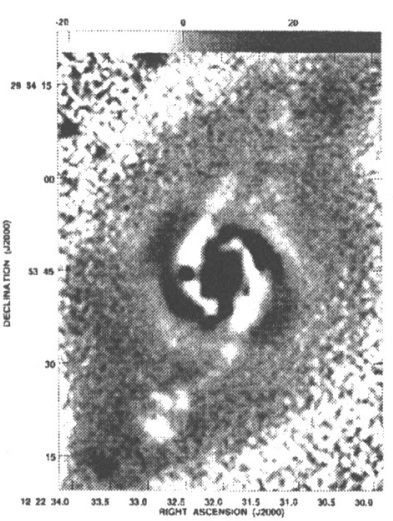

Figure 2. The structure of the galaxy NGC 4314 from an unsharp masked image. a) the differential structure from $B V I$ observations covering all of the primary stellar bar of the galaxy $\left(|r|<40^{\prime \prime}\right)$ where $r$ is the distance from the center of the system; $b$ ) the total differential structure of a model; c) the modelled central part. 


\subsection{Dynamical evolution of the structure of a system in the orbital plane of a binary nucleus.}

We follow the temporal evolution of these models (for each individual shell and for the ensemble of all shells - see, for example, Figs. 3, 4) and compare resulting structures with those observed in NGC 4314. Fig. 3 shows the dynamical evolution of the whole system during $P_{f}=T_{e v}=5 P_{o}$. Fig. 4 shows the changes in time the structure of the shell with the initial radius $R_{o}=0.75$ during the same time $P_{f}$. We find a combination of initial conditions and model parameters that produces the same structure and velocity field as that found in the galaxy NGC 4314 (Figs. 2a, b, c, Figs. 5a, b for the time $P_{f}=T_{e v}=4 P_{o}$ ).

Figs. 2a, b, c show that the modelled and observed galaxies contain very similar structures at the time $T_{e v}=4 P_{0}$ : inner dumbbell and external bars, a spiral around an inner bar, as well as spirals and rings around an external bar. Note that these structures exist only in the binary orbit's plane XY. The structure of this model in other coordinate planes is completely different at this time. In these two planes we obtain a central core and rings but no spiral structure. In the future the structure of the system changes noticably (see Fig. 3 ); spirals and rings now visible in NGC 4314 will soon decay, and new spirals and rings appear and disappear during the dynamical evolution of the system. In contrast, over the same time span a stable dumbbell bar remains in the center.

\subsection{Projection effects. The velocity field.}

We determine the influence of projections on this structure and determine the probability that the binary black hole's orbit lies parallel to the principal plane of NGC 4314.

In order to study this effect in detail, we carried out the following simple simulations. We define a plane $X, Y_{\text {sky }}$ tangent to the celestial sphere. We then varied the angles $\alpha, \phi$ and $\theta$ (describing the orientation of the original $\mathrm{XY}$-coordinate plane of the black hole binary orbit) with respect to this plane $X, Y_{s k y}$. The angle $\alpha$ is the rotation of the XY-plane around the axis Z; the angle $\phi$ is the rotation of this plane around the axis $Y$ and the angle $\theta$ is the rotation of the plane $X Y$ around the axis $\mathrm{X}$. We next fixed the projected coordinates and velocities of all particles of our model - the shell with $R=0.75$ - on this plane $X, Y_{s k y}$ at time $T_{e v}=4 P_{o}$ and generate slices through our model for different values of the angles $\alpha, \phi$ and $\theta$.

We find for different angles $\alpha$, galaxies with similar structures will have the same dynamics. Projection effects in $\phi$ and $\theta$ are very significant for values of these angles of more than $20^{\circ}$. We can conclude that for our model (and for NGC 4314) the angles $\phi$ and $\theta$ of inclination of the basic dynamical plane XY of the binary orbit with respect to the plane $X, Y_{s k y}$ should be no more than $20^{\circ}$.

We consider the distributions of rotation velocities of particles in the plane $X Y$ of the central binary's orbit as well as the Z-coordinates and $V_{z}$ velocities of particles; the velocities $V_{z}$ in this case approximate the radial velocities $V_{r}$ (see Figs. 5a, b). The rotation curve for our model is similar to that of Benedict et al. from CO observations in NGC 4314 (Benedict et al 1996a, Fig. 13).

Fig. $5 \mathrm{~b}$ shows the $V_{z}$ velocity distribution of particles from the shell with $R_{0}=0.75$ for the combination of angles: $\alpha=0, \theta=0, \phi=20^{\circ}$. We can see that this distribution of velocities correlates with the structure of our model; 

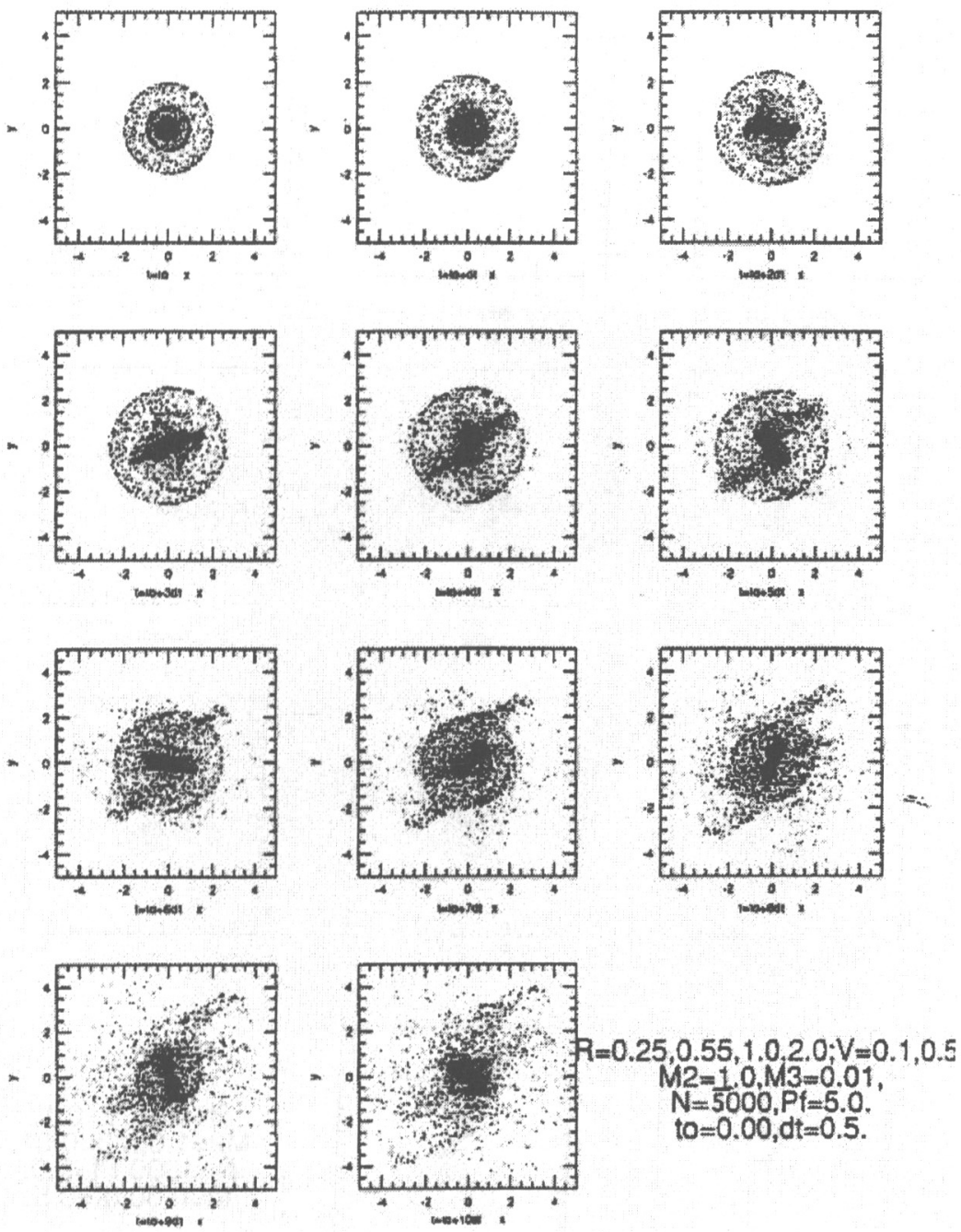

Figure 3. Dynamical evolution of an entire model with given parameters $R_{0}, V_{0}, M_{2}, N, T_{e v}$ for time $T_{e v}=5 P_{0}$, where $P_{0}$ is the period of rotation of the massive nuclear binary. These figures show 10 snapshots of the evolution of the structure over this time span. The scale is $\left[5 a_{0} \times 5 a_{0}\right]$ where $a$ is the semi-major axis of the massive nuclear binary. 

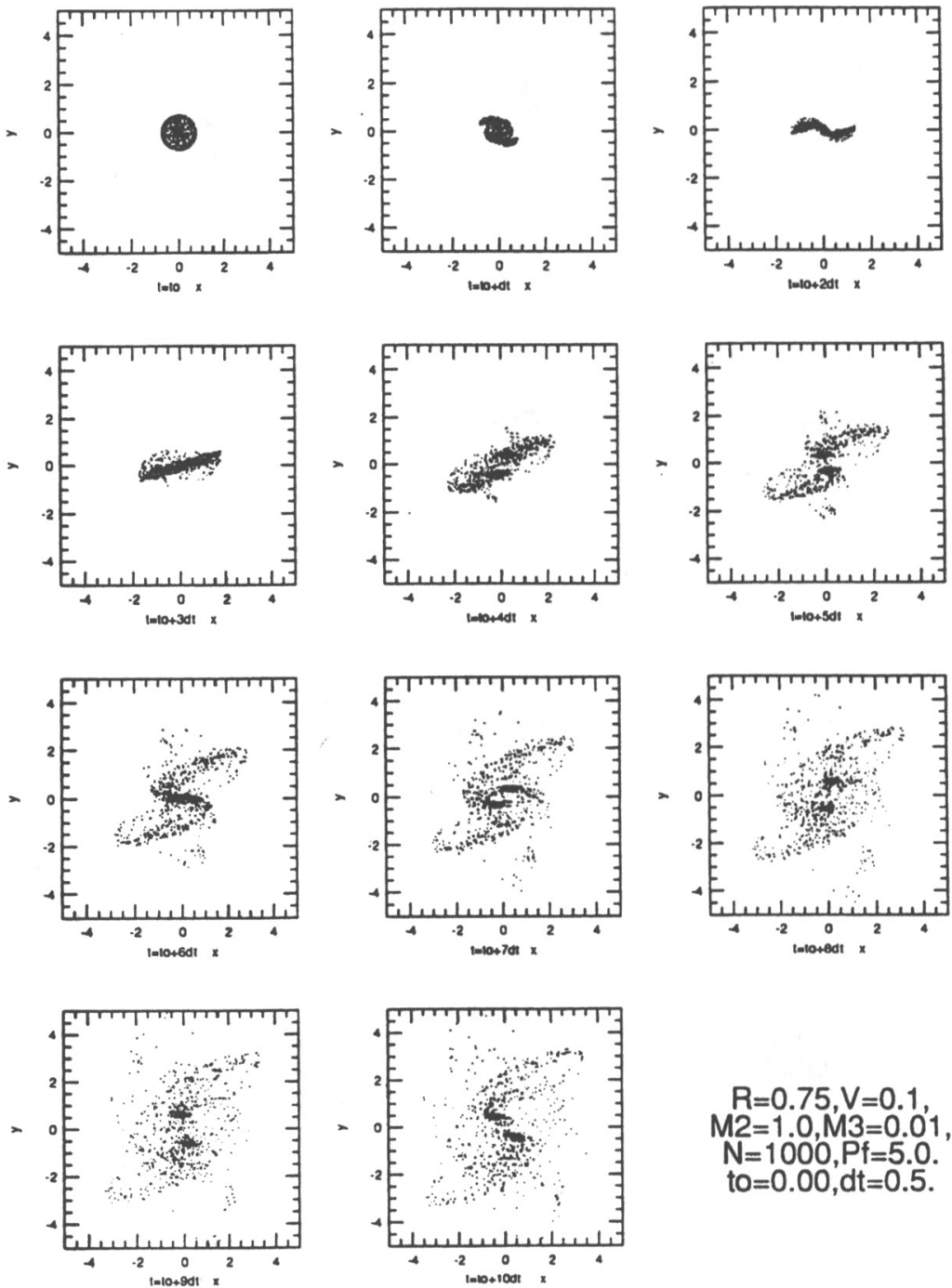

Figure 4. Dynamical evolution of the shell with $R_{0}=0.75$. Each figure presents 10 snapshots showing the evolution of the structure of these models to $T_{e v}=5 P_{0}$. The scale is the same as Fig 3 . 


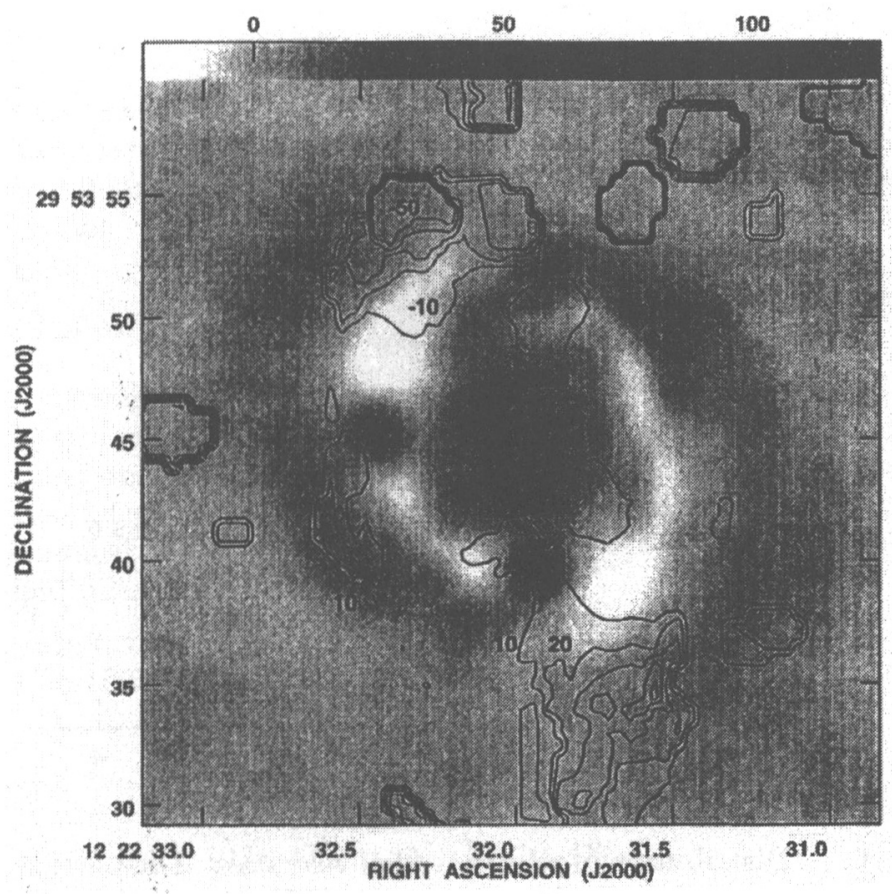

Figure 5. Distribution of relative radial velocities. CO residual map of NGC 3114 (Benedict et al. (1996a). 


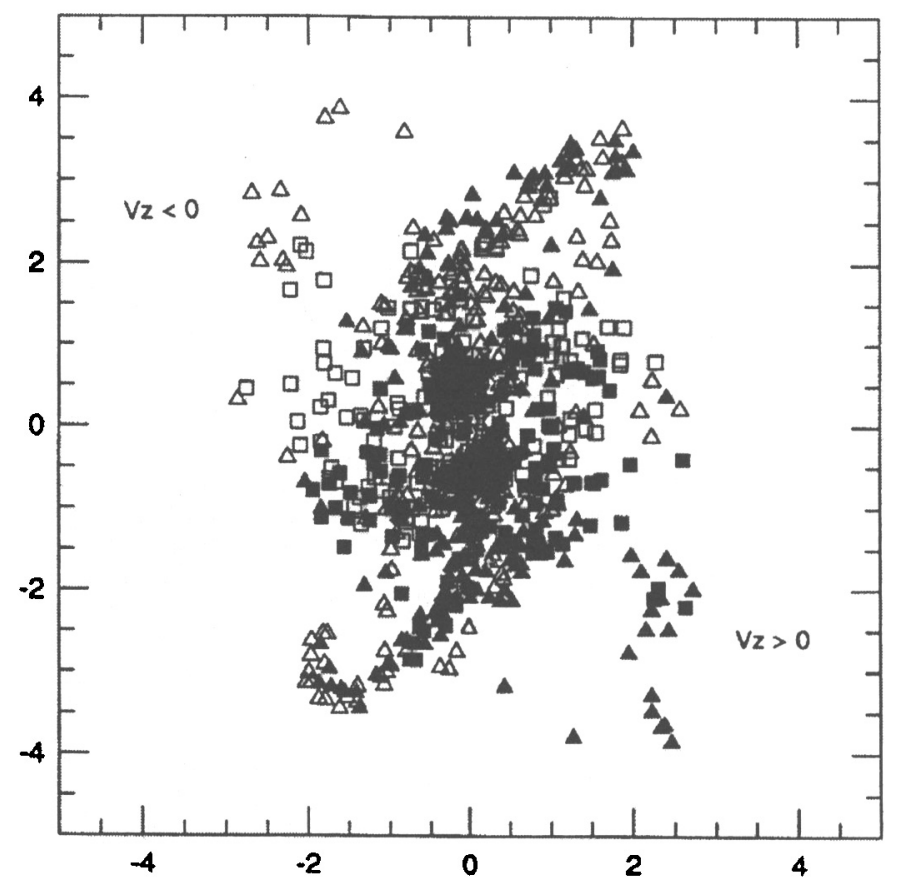

Figure 6. Distribution of relative radial velocities. The particle modelled radial $\left(V_{z}=V_{r}\right)$ velocity map in the XY-plane of the central binary for the parameters $R_{0}=0.75, V_{0}=0.1, M_{2}=1, N=1000, T_{e v}=$ $P_{f}=4.0$. The filled triangles and squares show the distribution of particles with positive radial velocities $\left(V_{Z}>0\right)$, the open triangles and squares show particles with negative radial velocities $\left(V_{Z}<0\right)$. The filled and open squares show particles with $\left|V_{Z}\right|>1$, and the filled and open triangles particles with $\left|V_{Z}\right|<1$ in our units. 
the modelled distribution $V_{z}$ is similar to the observed radial velocities $V_{r}$ in the galaxy NGC 4314 (Fig. 5a) In both cases, negative values of $V_{z}$ are found in the part of the figure with $x<0, y<0$ and $x<0, y>0$.

In contrast, for $\alpha=0, \theta=0, \phi=0^{\circ}$, we have a symmetrical distribution of the negative and positive 'radial' velocities $V_{z}$ of the particles; in case $\alpha=$ $0, \theta=20^{\circ}, \phi=0^{\circ}$ most $V_{z}$ are positive.

Therefore, we conclude that the structure of the galaxy NGC 4314 and the distribution of $V_{r}$ of CO can be successfully modeled if the main plane has angles close to $\theta=0$, and $\phi=20^{\circ}$.

We point out that:

1) much smaller $a_{o}$ and much larger $T_{e v}$ can produce similar morphologies. This allows us to hide the binary black hole in the existing nucleus;

2) the nuclear spiral appears only if the nucleus is presented with new particles (new gas);

3) this model can be extended to many more particles, many more shells with many different radii, and over far larger time periods with essentially unchanged results.

\section{Conclusion}

From our investigations we conclude that the evolution of a simple model of an N-body system containing a massive nuclear binary can generate structure similar to that observed in the galaxy NGC 4314 . We have determined an initial distribution of density and velocities of matter in the model, which over the time of the dynamical evolution, mimic structure observed in NGC 4314. We show the past and future evolution of this model.

Acknowledgments. This work partially supported by NASA HST GTO Grant NAG5-1603. The IAU is thanked for a travel grant to the meeting.

\section{References}

Aarseth, S., Zare K., 1974, Celest. Mech., 10, 185

Agekian, T., Anosova J., 1967. Sov. Astron. J., 44, 1267

Agekian, T., Anosova J., 1991. Sov. Astron. J., 68, 1099

Anosova, J., 1986, Astrophys. Space Sci., 124, 217

Anosova, J., 1991. Celest. Mech. \&Dynam. Astron., 51, 1

Anosova, J. and Zavalov. 1989, Sov. Astron. J., 66, 152

Anosova, J. and Anandarao B. 1994, Astrophys. Space Sci., 220, 83

Anosova, J. and Tanikawa K. 1995, Astrophys. Space Sci., 234, 191

Basu, D., Valtonen, M. J., Valtonen, H. and Mikkola,S. 1993, Astron. Astroph. 272,417

Benedict, G. F et al. 1992, AJ, 103, 757

Benedict, G. F et al. 1993, AJ, 105, 1369

Benedict, G. F., Smith B. J., Kenney J. D. P., 1996a, AJ, 111, 1861

Benedict et al. 1996b, BAAS, 28, 1187 
Binney J., Tremaine S. 1987, Galactic Dynamics, Princeton, Princ. Univ. Press Combes F., 1993, In: "Physics of Galaxies: Nature or Nurture". Ed. Balkowski C., $\mathrm{p} 137$

Lin, D. N. C. and Saslaw, W. C., 1977, ApJ, 217, 958

Lin, D. N. C. and Lynden-Bell, D., 1982, MNRAS, 198, 707

Murai,T. and Fujimoto, M., 1980, Publ. Astron. Soc. Japan, 32, 581

Sellwood J. A. 1987, ARA\&A, 25, 151

Sellwood J. A. 1993, Rep. Prog. Phys., 56, 173

Toomre A. and Toomre J. 1972, ApJ, 178, 623

Valtonen, M. J. and Byrd, G. G., 1979, ApJ, 230, 655

Valtonen, M. J. and Byrd, G. G., 1986, ApJ, 303, 523

Valtonen, M. J. et al., 1984a, A\&A, 143, 182

Valtonen, M. J. et al., 1984b, ApSS, 107, 209

Valtonen, M. J. et al., 1985, A\&A, 143, 182

Valtonen, M. J., 1988, Vistas Astron., 32, 23

Yoshiaki, T., Keiichi, W. 1996, ApJ, 469, 581 